\title{
Individual differences in processing stimulus dimensions: Relation to selective processing abilities
}

\author{
THOMAS B. WARD \\ Texas A\&M University, College Station, Texas
}

\begin{abstract}
Three studies investigated individual differences in the tendency to process stimulus dimensions in a manner that is either primarily separable or integral. All employed a restricted classification task that allows participants to classify items on the basis of component dimensions (separable perception) or overall similarity (integral perception). Experiment 1 revealed that individual differences in the tendency to classify by dimensional structure versus similarity generalize to two types of stimuli (separate and moderately separable) which were thought to require different types of processing for the perception of their component dimensions. The study also indicated that individuals differ in their tendencies to increase the use of dimensional structure with more exposure to the stimuli. Experiment 2 revealed that both overall dimensional responding and the tendency to increase such responding with exposure to the stimuli were positively related to the ability to break stimuli into components, as measured by Embedded Figures Test performance. Experiment 3 also showed a relation between increased dimensional responding and performance on another selective processing task. The results are discussed in terms of their implications for understanding both individual differences in the processing of stimulus dimensions and the separable-integral perception distinction.
\end{abstract}

Specification of the nature of psychological or perceived stimuli presents a fundamental problem for psychological theory and research. Implied in this statement is the idea that the nature of the stimulus must be specified from the point of view of the perceiver and may not simply be an arbitrary description of how the experimenter constructed the stimuli. This is so because, in order to be complete, theories of cognition and perception must address not only the processes thought to be involved, but also the nature of the stimuli being processed, how different types of stimuli lead to differing psychological representations, and how those different representations affect the individual's ability to apply certain processes.

One important approach to specifying the nature of the perceived stimulus and how that nature can affect processing is found in Garner's work $(1974,1976,1983)$ on different types of stimulus dimensions. In an integrative discussion of his own work and that of other investigators (see, e.g., Lockhead, 1966; Shepard, 1964), Garner (1974) noted that the dimensions that psychologists use to construct stimuli can combine or interact in several different ways and that those different dimensional interactions can have differing perceptual and processing consequences.

Portions of these data were presented at the meeting of the Midwestern Psychological Association, Chicago, May 1983. The author thanks Jorge Mendoza for statistical advice, Colleen Foley for collecting the data for Experiment 3, and two anonymous reviewers for helpful comments on an earlier version of this paper. Requests for reprints should be sent to Thomas B. Ward, Department of Psychology, Texas A\&M University, College Station, TX 77843.
Perhaps the greatest amount of research on different dimensional combinations has been stimulated by Garner's (1974) theoretical account of the difference between separable and integral dimensions. Separable dimensions (e.g. , the size and brightness of geometric forms) are perceived (i.e., represented psychologically) as separate or isolated from one another and can be processed independently. When presented with a stimulus composed from separable dimensions, an individual can readily attend to one dimension (e.g., size) and ignore the other (e.g., brightness). In contrast, integral dimensions (e.g., hue and saturation) are perceived in a unitary way. When presented with a stimulus composed from integral dimensions, the individual would have great difficulty in selectively attending to one dimension (e.g., hue) and ignoring the other (e.g., saturation). Thus, there appear to be both perceptual (separate vs. unitary) and processing (e.g., selective attention) consequences of the differing dimensional combinations.

From the above description, it should be clear that the separable-integral distinction has been viewed primarily as a stimulus concept. Different types of physical stimuli are thought to lead to differing psychological representations. Dimensions themselves are characterized as separable or integral, and presumably they should be perceived as such by all observers.

Despite the fact that this approach to characterizing different types of stimuli and their effects on processing has been extremely valuable, it is becoming increasingly clear that there are large and reliable differences in the way that different people perceive and process "separ- 
able" stimuli. That is, the very same physical stimulus appears to be a different psychological stimulus for different individuals. For example, young childen give responses indicative of integral perception when presented with stimuli which are separable for adults (Shepp \& Swartz, 1976; L. B. Smith \& Kemler, 1977; Ward, 1980). In addition, older retarded (Kemler, 1982) and hyperactive (Fisher, 1977) children tend to give the integral-type responses more characteristic of younger nonretarded and nonhyperactive children. Finally, even among adults of approximately the same age and ability level, there appear to be consistent individual differences in the tendency to give primarily separable or integral responses to certain stimuli (J. D. Smith \& Baron, 1981; Ward, 1983). Thus, the task of specifying the nature of the psychological stimulus becomes even more complex. We must consider not only how different physical dimensions, in a general way, lead to differing psychological representations and processing consequences, but also how those psychological representations and consequences may differ for particular individuals.

In view of the above considerations, the research reported in the present paper was designed to extend our understanding of adult individual differences in the tendency to perceive or process stimuli in a primarily integral or separable manner. Three major issues were addressed. First, the studies examined the generality of those differences across different types of stimuli. Clearly, the more those individual differences generalize to different types of stimuli, the more important they become. Second, the studies examined the extent to which individuals come to the task with already established different modes of processing versus the extent to which individuals develop those styles with experience in the task. Finally, the studies examined the relation of those individual differences to the ability to process information in a selective manner.

This last issue is particularly important. Among the more important contributions of Garner's (1974) work is that it demonstrates (1) that the nature of the perceived stimulus greatly affects processing, and, therefore, (2) that information processing models must specify not just processes, but also how processing options are constrained by the nature of the stimuli. Thus, if adopted, Garner's suggestions represent a significant advance in the sophistication of information processing models. Similarly, individual differences in the tendency to perceive more separably or integrally would be of utmost importance if it were demonstrated that those differences were related to the individual's ability to process information in a particular way. The implication would be that processing models would have to specify not just the processing constraints imposed by different types of stimuli, but also how processing options differ for individuals with differing perceptual styles.

The task used throughout these studies is called "restricted classification," and it makes use of Garner's (1974) notion that the relations among separable stimuli are perceived in terms of a dimensional structure, whereas the relations among integral stimuli are perceived in terms of overall similarity. In the restricted classification task, research participants are shown triads of stimuli and are asked to indicate the two out of three that go together best. Triads are constructed so that classifications made on the basis of similarity are pitted against those made on the basis of identity along one dimension. If the participant consistently uses similarity in classifying stimuli (similarity responses), the result indicates integral perception. If the participant classifies on the basis of identity along one dimenson (dimensional response), the result indicates separable perception (Garner, 1974; L. B. Smith \& Kemler, 1977; Ward, 1980, 1983).

\section{EXPERIMENT 1}

If there is a general tendency to represent the world in an integral or separable fashion, then an individual's responding should be consistent across many different types of dimensions. Using a task similar to restricted classification, J. D. Smith and Baron (1981) found significant positive correlations between the proportion of similarity responses given by participants to both auditory and visual stimuli. The dimensions used by J. D. Smith and Baron appear to have been separable and integral. Thus, there is good evidence for the generality of individual differences in separable and integral responding. In the present study, an attempt was made to extend the generality of such differences.

The dimensions used in the present study were length and density, which have yielded a pattern of moderately separable responding (Ward 1978, 1980, 1983), and circle size and angle of radius orientation, which have been described as being "separate" dimensions (Burns, Shepp, McDonough, \& Wiener-Erlich, 1978). In contrast to other types of stimuli that require analysis for the perception of their dimensional structure (e.g., length and density; see Ward, 1983), separate dimensions are thought to yield their dimensional structures automatically (Burns et al., 1978). Although the evidence for such automatic access is suggestive rather than definitive (see Burns et al., 1978), at a purely descriptive level, the circle-size/radiusorientation stimuli differ from those used in previous studies of individual differences in separable-integral responding. The area of a circle and the angle of orientation of a line are physically separable. The presence of one dimension does not logically imply the existence of the other. Circles can vary in area without containing radii at all. Lines may vary in orientation with respect to vertical without existing inside circles. ${ }^{1}$ Other stimuli that have been used (e.g., angle and side length, size and brightness, length and density) are not physically separable in the same sense. For angles to vary in degree, those angles must have sides of some length; for a form to have some brightness, that form must exist and therefore have some size; and so on.

If the analysis requirements for the length-density and circle-size/radius-orientation stimuli are different, it might be expected that individuals will not show consistent pat- 
terns of responding to these two different types of dimensions. On the other hand, if there is a general tendency to perceive in a separable or integral manner, there should be positive correlations between the number of similarity responses given to the moderately separable stimuli and the number given to the separate stimuli and also between the numbers of dimensional responses given to those stimuli.

Perhaps more important than the issue of generality, a second issue addressed in this study is whether or not there are individual differences in the tendency to increase dimensional responding with experience in the task. Ward (1980) found that adults were more likely than children to show such an increase, and attributed the difference to the adults' greater perceptual learning ability. It is reasonable to suppose that individual differences among adults will mimic that developmental finding. Therefore, it might be expected that individuals who show an increase in dimensional responding to one pair of dimensions (e.g., length and density) will be more likely to show an increase in dimensional responding to the other pair of dimensions (circle size and radius orientation). Information on this issue is lacking in previous studies of individual differences in responding to stimulus dimensions.

Examination of individual differences in the tendency to increase dimensional responding is important because the presence of such differences could affect the way in which we conceptualize the overall individual differences that have been found. If some individuals show increases in dimensional responses across both sets of dimensions whereas others show no such increases, then at least some of the previously observed individual differences in overall performance might be related to perceptual learning ability or the ability to extract dimensional information. In fact, it could be that all individuals come to the task with basically the same response tendencies but that some learn about the dimensional structure of the stimuli and shift to dimensional responding whereas others do not. In this case, the individual differences in performance would be more reasonably thought of as reflecting perceptual learning ability rather than a general separable or integral processing style. Basically, the question is whether individuals come to the task with different styles of processing dimensions or develop those styles only with experience in a particular laboratory task. In the former case, correlations between the various response types should hold both at the beginning and at the end of the task, whereas in the latter case correlations should emerge only later in the task. Since responding was observed across trial blocks, changes in correlations with experience in the task could be observed.

\section{Method}

Research participants. Ninety undergraduates enrolled in introductory psychology courses at Texas A\&M University participated in order to fulfill a course requirement.

Stimuli. The stimuli were triads composed of lines of dots that varied in line length and dot density or circles with various sizes and angles of orientation of radius. For the length and density items, the lines for a given triad were chosen from the set of lines represent- ing the possible combinations of lengths $1,1.5,2,3,4,6$, and $8 \mathrm{~cm}$ and of densities (in terms of interdot distance) of $1.5,1, .75, .50$, $.37, .25$, and $.125 \mathrm{~cm}$. Twelve triads were constructed using similarity-scaling data (Ward, 1978) to pit classifications made on the basis of dimensional identity against those made on the basis of overall similarity. Six of the triads had two members that were identical in length and very different in density, and six had two members that were identical in density and very different in length. ${ }^{2}$ The members of a triad that were identical along one dimension and very different on the other will be referred to as "dimension pairs." All triads had a third member, which differed from the other two on both dimensions. The third member differed slightly (generally by only one level on each dimension) from one of the other members, and this combination of items is referred to as a "similarity pair." For all triads, the similarity pair had been rated as more similar than the dimension pair (Ward, 1978). Examples of length and density triads are depicted in Figures $1 \mathrm{a}$ and $1 \mathrm{~b}$, respectively.

The lines of each triad were typed onto index cards horizontally and centered one above the other. Three versions of each triad were typed so that each line appeared once in each position on the card (top, middle, bottom) and each possible pairing of lines in the triad appeared once in each possible combination of positions (top-middle, middle-bottom, top-bottom). The index cards were photographed so that triads could be presented by a slide projector.

Circle triads were constructed on the basis of data reported by Burns et al. (1978) in order to pit classifications made on the basis of dimensional identity against those made on the basis of overall similarity. The circles were white on a gray background, and each had a black line as a radius that extended $8 \mathrm{~mm}$ from the center of the circle. Circles for a given triad were chosen from the set of possible combinations of sizes $4.5,6.4,9.0,12.8,18.1$, and $25.8 \mathrm{~cm}^{2}$ and radius orientation of $5^{\circ}, 35^{\circ}, 65^{\circ}, 95^{\circ}, 125^{\circ}$, and $155^{\circ}$ from vertical. Six triads had two members that were identical in size and very different in radius orientation, and six had two members that were identical in orientation and very different in size. As with the length and density triads, these will be referred to as the dimension pairs of a triad. All triads had a third member which
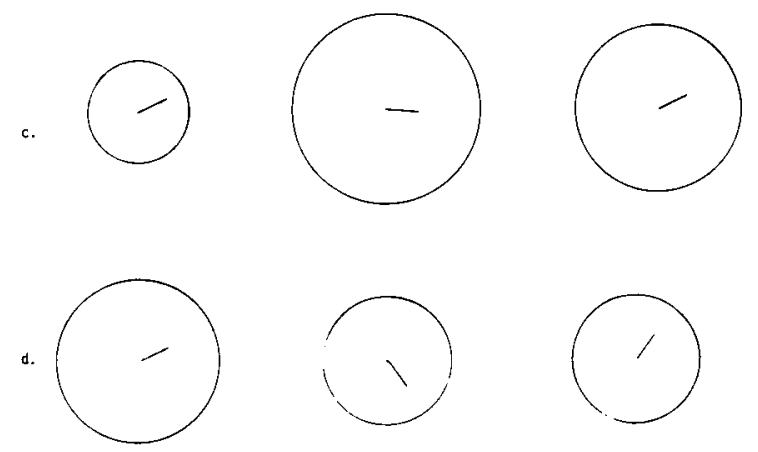

Figure 1. Schematic representations of (a) length, (b) density, (c) orientation, and (d) circle size triads used in Experiment 1. The dimension pairs are, respectively, in the top/middle, top/bottom, left/right, and middle/right positions for the length, density, orientation, and size triads shown in this figure. The respective positions for the similarity pairs are middle/bottom, top/middle, middle/right, and left/right for the particular triads shown. Position of response type was counterbalanced across trial blocks for each triad. 
differed slightly (generally by one level) from one of the other members along both dimensions, and this combination is referred to as the similarity pair in the triad. As nearly as could be determined from the Burns et al. data, the similarity pair for each triad was more similar than the dimension pair for that triad.

The circles of each triad were arranged horizontally and photographed. Three versions of each triad were constructed such that each item appeared once in each position (left, middle, right) and each possible pairing of circles appeared once in each combination of positions (left-middle, left-right, middle-right). Schematic representations of orientation and size triads are depicted in Figures $1 \mathrm{c}$ and $1 \mathrm{~d}$, respectively.

Procedure. All participants were shown both the length-density and circle-size/radius-orientation triads. Half of the participants viewed all three versions of each of the 12 length-density triads and then all three versions of each of the 12 circle triads. For the other half of the participants, this order of presentation was reversed. For both the length-density items and the circles, presentations were structured such that all 12 triads appeared in a different random order in each of three trial blocks (trials). Triads were presented for $8 \mathrm{sec}$ each.

Participants were instructed to pick the two out of the three items in each triad that went together best. For the length-density triads, the top, middle, and bottom positions on the slide were given the labels $a, b$, and c, respectively. For the circles, the left, middle, and right positions were assigned the labels $a, b$, and $c$, respectively. The participants indicated their choices by writing, on response forms, the letters for the pair of their choice.

For each triad, three types of responses were possible. Participants could choose the dimension pair as the ones that went together best. This was called a dimensional response, and it indicates separable perception. Alternatively, participants could choose the similarity pair. This was labeled a similarity response, and it indicates integral perception. Finally, the participants could choose the pair that was neither identical along one dimension nor overall most similar, and this is referred to as a haphazard response.

\section{Results and Discussion}

Overall response patterns. Table 1 presents the number (and standard deviation) of dimensional, similarity, and haphazard responses for the length-density and circlesize/radius-orientation stimuli across Trial Blocks 1-3 as

Table 1

Number (and Standard Deviation) of Dimensional, Similarity, and Haphazard Responses to the Length-Density (Dots) and Size/Radius-Orientation (Circles) Items and Correlations Between Those Responses

\begin{tabular}{llcccc}
\hline & & \multicolumn{4}{c}{ Trial Block } \\
\cline { 3 - 6 } Response & Dimension & 1 & 2 & 3 & Total \\
\hline Dimensional & Dots & 6.52 & 6.78 & 7.39 & 20.59 \\
& & $(2.45)$ & $(3.18)$ & $(3.07)$ & $(7.99)$ \\
& Circles & 5.48 & 7.17 & 7.18 & 19.82 \\
& & $(2.52)$ & $(2.62)$ & $(2.54)$ & $(6.79)$ \\
& $\mathrm{r}$ & $.35^{* *}$ & $.42^{* *}$ & $.50^{* *}$ & $.50^{* *}$ \\
Similarity & Dots & 4.66 & 4.68 & 3.83 & 13.17 \\
& & $(2.19)$ & $(3.01)$ & $(2.84)$ & $(7.34)$ \\
& Circles & 5.53 & 4.21 & 4.12 & 13.87 \\
& & $(2.44)$ & $(2.67)$ & $(2.45)$ & $(6.70)$ \\
Haphazard & Dots & $.30^{* *}$ & $.46^{* *}$ & $.54 * *$ & $.53^{* *}$ \\
& & .82 & .64 & .78 & 2.24 \\
& Circles & $.96)$ & $(.86)$ & $(.93)$ & $(1.86)$ \\
& & $(1.08)$ & .62 & .70 & 2.31 \\
& $\mathrm{r}$ & $.19 *$ & .06 & .11 & $(1.69)$ \\
& & & & & .08 \\
\hline
\end{tabular}

$\overline{* p}<.05 . \quad{ }^{* * p}<.01$. well as the total number of such responses. As can be seen in the last column of Table 1, the overall pattern of responding to the two different types of dimensions was roughly equivalent. There were no significant differences between the number of dimensional $[F(1,86)=2.03$, $\mathrm{p}>.15]$, similarity $[\mathrm{F}(1,86)=2.46, \mathrm{p}>.12]$, or haphazard $(F<1)$ responses given to the two different types of stimuli. For both types of dimensions, the predominant mode of classification was dimensional and there were significantly more dimensional responses than either similarity $[F(1,86)=29.83, p<.001$, and $F(1,86)=$ $19.67, \mathrm{p}<.001]$ or haphazard $[\mathrm{F}(1,86=340.50$, $\mathrm{p}<.001$, and $\mathrm{F}(1,86)=456.68, \mathrm{p}<.001]$ responses for the length-density and circle items, respectively. There were also significantly more similarity than haphazard responses for the length-density $[F(1,86)=143.08$, $\mathrm{p}<.001]$ and circle $[\mathrm{F}(1,86)=174.97, \mathrm{p}<.001]$ items.

The analysis on length-density items revealed an effect of triad type $[\mathrm{F}(1,86)=30.80, \mathrm{p}<.001]$, which indicates a preference to classify on the basis of density over length. The mean number (and standard deviation) of dimensional responses was 12.41 (5.00) and 7.96 (5.75) for the dimensions of density and length, respectively. The result is consistent with the results of previous research (Ward, 1980, 1983) and indicates that the dimension of density is more potent, or salient, than the dimension of length. None of the above analyses revealed significant effects of sex of the participant.

Of greater importance than the number of each type of response to each type of stimulus is the relation between the responses. The last column of Table 1 also shows the correlations between the number of each type of response to the length-density stimuli and the number to the circlesize/radius-orientation stimuli. As can be seen, there were significant positive correlations between the numbers of dimensional and similarity responses given to the two types of stimuli. Thus, individual differences in the tendency to process information in a primarily separable or integral manner appear to extend to moderately separable and "separate" dimensions.

Changes in responding over trials. As can be seen in Table 1 , for both the length-density items $[F(2,174)$ $=11.35, \mathrm{p}<.001]$ and the circle items $[\mathrm{F}(2,174)=$ $45.20, \mathrm{p}<.001$ ], there was a significant increase in dimensional responding across trial blocks. This shift in response tendency could reflect a type of perceptual learning in which participants discover either the dimensional structure of the stimuli or the usefulness of that structure for classifying the items.

It is unlikely that the increase in dimensional responding simply represents a shift away from random responding or a generalized adjustment to the testing situation. As can be seen in Table 1, there was virtually no change in the number of haphazard responses across trial blocks for either the length-density or the circle materials. Rather, the increase in dimensional responding appears to represent a shift from reliance on one sensible type of infor- 
mation (similarity) to greater reliance on another type (dimensional identity) with experience. As indicated in Table 1, for both length-density $[F(2,174)=11.97$, $p<.01]$ and circle items $[F(2,174)=25.98, p<.001]$, there was a significant decline in similarity responding across trial blocks.

An important question is whether individuals differ in the tendency to show an increase in dimensional responding. The question can be asked in at least two ways. The first is whether or not individuals who show increased dimensional responding to one set of materials are more likely than other individuals to show increased dimensional responding to the other set of materials. The second is whether or not the magnitude of the change in response to one set of materials is positively related to the magnitude of the change in response to the other set of materials. The former question is best addressed via a chisquare analysis; the latter is best addressed by computing a correlation coefficient. The chi-square approach has the advantage that it is unnecessary to assume that the magnitude of the increase in dimensional responding to one set of materials is linearly related to the magnitude of the increase in dimensional responding to the other set of materials. An increase of $x$ units of dimensional responding to length and density may or may not be equivalent to the same-size increase in dimensional responding to size and radius orientation. The advantage of a correlational approach is that it can determine whether or not a particular type of relation (i.e., linear) exists. Given the limited state of knowledge regarding increases in dimensional responding, it may be best initially to make as few assumptions as possible about the nature of any existing relations.

In order to conduct a chi-square analysis, change scores (number of dimensional responses on Trial Block 3 minus number of dimensional responses on Trial Block 1) were used in a categorical manner (positive, which indicated increased dimensional responding, vs. zero or negative, which indicated no change or decreased dimensional responding). These categorical change scores were used to classify individuals into one of four cells of a $2 \times 2$ contingency table based on whether or not they showed an increase, from Trial Block 1 to Trial Block 3, in dimensional responding to the length-density and/or circle items. Of the 90 participants, 42 showed an increase in dimensional responding to both types of stimuli, 7 showed increased dimensional responding to length and density but not to the circles, 27 showed increased dimensional responding to the circles but not to length and density, and 14 showed no increase in dimensional responding to either type of stimulus material. Note that this approach results in each participant's contributing to one and only one cell in the $2 \times 2$ table. A chi-square test on these contingencytable classifications indicated that the classifications were not independent of one another $\left[\chi^{2}(1)=4.92, p<.05\right]$. The result indicates that participants who showed an increase in dimensional responses to one of the pairs of dimensions (e.g., length and density) were more likely to show an increase in dimensional responses to the other pair of dimensions (e.g., circle size and radius orientation). Thus, individual differences in processing dimensional information are not limited to the overall tendency to use either primarily separable or primarily integral information. It appears that individuals also differ in their tendencies to shift toward greater use of dimensional information with exposure to the dimensions concerned.

To determine if the magnitude of the increase in dimensional responding to one set of materials was related in a linear way to the magnitude of the increase in dimensional responding to the other set, a correlation coefficient was computed between the change scores (Trial Block 3 minus Trial Block 1) for the length-density and circle items. The overall correlation approached, but did not quite reach, conventional levels of statistical significance $(\mathrm{r}=.146, \mathrm{p}=.085)$. However, a potential problem with this overall correlation approach is that the amount of increase in dimensional responding that any individual can show is related to that person's initial level of dimensional responding to that set of materials (Trial Block 1). If some participants started at high levels of dimensional responding to one set of materials and lower levels of dimensional responding to the other set, the greater room for increase with the latter set would work against finding a significant correlation between the magnitudes of the increases. Therefore, the correlation was adjusted for the number of dimensional responses given to each type of stimulus on Trial Block 1. The resulting partial correlation was significant $(r=.285, p<.01)$. Thus, the results of the partial correlational analysis are consistent with those obtained using the chi-square aproach. When controlling for initial level of responding, there is a significant linear relation between the magnitudes of the increases shown to the two types of materials.

Given that some individuals appear to show consistent increases in the use of dimensional information with experience in the task, it could be that individuals develop their styles in response to task demands rather than come to the task with those styles already established. To check on this possibility, correlations on the number of dimensional responses given to the two types of stimuli were computed separately for Trial Blocks 1,2 , and 3 . Although, as shown in Table 1, the correlations increased across trial blocks, the increase was not significant and the correlations in each trial block were significant at the .001 level. Thus, there do seem to be individual differences in processing from the very beginning of the task. This observation, combined with the slight, though nonsignificant, increase in correlations over trial blocks, might indicate that individuals come to the task with generally separable or integral styles of processing and refine those styles in relation to the specific task encountered.

\section{EXPERIMENT 2}

The results of Experiment 1 extend our knowledge of individual differences in the processing of dimensional information in two ways. First, they indicate that the 
differences generalize to two types of dimensions which had not been investigated previously, and which theoretically might require different amounts of analysis for the perception of their dimensional structure (see Burns et al., 1978; Ward, 1983). Second, the results suggest that individual differences in processing dimensional stimuli may extend to the tendency to shift to higher levels of dimensional responding with experience in the restricted classification task. The latter result is particularly important in that it highlights the need to consider such differences when we investigate the relation between separable-integral responding and other measures of performance.

A crucial question to be asked is whether or not the differences noted above really make any difference. If the differences are confined to the way in which individuals perform in restricted classification tasks, then knowledge of such differences may be of limited utility. The differences could simply reflect differences in responsiveness to the instructions or demands of a particular task. On the other hand, if the differences reflect something more basic about the nature of the individual's mode of processing and perceiving the world, then they become important. To the extent that individual differences in restricted classification task performance correlate with performance in other perceptual and cognitive tasks, we have evidence that such restricted classification task performance does reflect basic underlying cognitive/perceptual differences rather than differential responding to one particular task. We also begin to understand more about the nature of those differences.

Although the possible relations between separableintegral processing in the restricted classification task and other measures of processing are manifold, Garner's (1974) theoretical account of separable versus integral perception can be used as a starting point for the generation of predictions. This approach was followed in the second experiment, which was designed to determine whether the tendency to classify on the basis of dimensions versus similarity was related to the ability to extract relevant information from a background of irrelevant information. Since both similarity responding and difficulty in selective attention characterize integral perception (Garner, 1974), it is reasonable to expect that individuals who give many similarity classifications are "integral" perceivers who have difficulty separating relevant and irrelevant information. That is, individuals might give many similarity responses because they are less able than dimensional responders to break stimuli down into component dimensions in order to classify them. J. D. Smith and Baron (1981), on the other hand, suggest that differences in performance on the restricted classification task simply reflect preferences for classifying items according to particular rules. They note that there was no relation between their participants' performance in restricted classification tasks and their performance on selective attention tasks using the same dimensions, and that performance on the latter, but not on the former, was correlated with other measures of ability (e.g., intelligence test scores). They suggest that the restricted classification and speeded sorting tasks may tap two different types of individual differences.

In order to investigate this issue further, the Group Embedded Figures Test (GEFT) (Oltman, Raskin, \& Witkin, 1971) was used to divide participants into those with greater and those with lesser ability to break stimuli into components. These groups were then compared in terms of their performance (similarity vs. dimensional classifications) on a restricted classification task. If selective processing and restricted classification tasks tap separate individual differences, then performance on the two tasks should be unrelated. If classifying tendencies and selective processing ability are related, then individuals showing good GEFT performance should be the same individuals who give many separable (dimensional) responses. ${ }^{3}$

An additional issue addressed in the second experiment was whether the individual differences in the tendency to shift toward more dimensional responses across trials, as found in Experiment 1, was related to GEFT performance. Finding such a relation would add support to the tentative conclusion of Experiment 1 that individuals differ in the tendency to show increased dimensional responding, and would illustrate the importance of considering such differences when studying separable and integral responding. If the shift toward dimensional responding is related to the ability to extract task-relevant information (e.g., dimensions for classifying stimuli), then individuals with greater ability to extract the relevant simple figures embedded in irrelevant backgrounds should be more likely to show such a shift.

\section{Method}

Research participants. Two hundred and four undergraduates enrolled in introductory psychology classes at Texas A\&M University participated in order to fulfill a course requirement.

Stimuli. The stimuli used in the restricted classification task were the triads composed of lines of dots varying in line length and dot density described in Experiment 1. The embedded figures were those in the standard GEFT booklets (Oltman et al., 1971).

Procedure. All participants were shown the length-density triads and then given the GEFT. The procedures for the restricted classification task were as described in Experiment 1. The GEFT was administered according to standard procedures for that task.

\section{Results and Discussion}

Scores on the GEFT were used to divide participants into groups manifesting greater and lesser ability to extract relevant figures from an irrelevant background. Using medians supplied by Witkin, Oltman, Raskin, and Karp (1971), a score of 12 (out of a possible 18) was used as a dividing point, such that those scoring 12 and below were assigned to the low-ability group and those scoring 13 and above were assigned to the high-ability group. This resulted in 100 participants in the low group and 104 in the high group. To produce equal Ns, four participants were randomly eliminated from the high group. Quite fortuitously, there were 64 females and 36 males in each of the resultant groups.

The number (and standard deviation) of dimensional responses for the high and low GEFT ability groups is 
Table 2

Number (and Standard Deviation) of Dimensional Respnses for High and Low GEFT Ability Groups in Trial Blocks 1, 2, and 3

\begin{tabular}{lccc}
\hline & \multicolumn{3}{c}{ Trial Block } \\
\cline { 2 - 4 } Group & 1 & 2 & 3 \\
\hline High & 6.86 & 7.03 & 7.80 \\
& $(2.29)$ & $(2.88)$ & $(2.89)$ \\
Low & 6.35 & 6.00 & 6.39 \\
& $(2.03)$ & $(2.50)$ & $(2.96)$ \\
\hline
\end{tabular}

shown in Table 2 separately for Trial Blocks 1, 2, and 3. An analysis of variance was performed on the number of dimensional (separable) responses, using GEFT ability (high vs. low) and sex as between-subjects variables and trial blocks and triad type (length vs. density) as within-subject variables. The only significant effects in the analysis were ability $[F(1,198)=9.10, \mathrm{p}<.01]$, trial blocks $[\mathrm{F}(2,396)=8.49, \mathrm{p}<.001]$, triad type $[F(1,198)=97.58, p<.001]$, and the ability $\times$ trials interaction $[F(2,396)=4.45, p<.05]$. Basically, as can be seen in Table 2, individuals with greater GEFT ability made a higher proportion of dimensional responses; participants shifted to more of those responses over trial blocks, and high GEFT performers were more likely to show that shift. The trial blocks $\times$ ability group interaction was examined further by way of pairwise comparisons using the Scheffe procedure to control the Type I error rate across all comparisons. Since the procedure is an extremely conservative one, a significance level of .10 was used to calculate the critical $F$ ratio for pairwise comparisons. Using the 2 and 396 degrees of freedom associated with the trial blocks $\times$ ability group interaction, an $\mathrm{F}$ ratio of 2.30 is needed to achieve significance at the .10 level. Thus, the critical $F$ ratio for the pairwise comparisons relevant to that interaction is (trials -1$) \times$ (groups -1 ) $\times 2.30$ or 4.60 . In comparison to that critical $F$ ratio, the increase shown by the high GEFT ability group was significant for Trial Block 1 versus Trial Block $3[\mathrm{~F}(1,396)=19.24]$ and Trial Block 2 versus Trial Block $3[\mathrm{~F}(1,396)=12.91]$. There was no significant change in responding for the low GEFT ability group $(F<1$ in all cases). Applying the same critical $F$ ratio and using a compromise error term (see Winer, 1971, p. 545), pairwise comparisons revealed that the high GEFT group made significantly more dimensional responses than the low-ability group on Trial Blocks $2[\mathrm{~F}(1,396)=7.75]$ and $3[F(1,396)=14.52]$, but not on Trial Block 1 $[F(1,396)=1.90]$. This last finding illustrates the usefulness of considering a shift toward dimensional responding when studying individual differences in separableintegral responding. It appears that the tendency to increase dimensional responding is a major determinant of the overall separable-integral response differences found between the high and low GEFT ability groups.

Although it would be difficult to theoretically justify a planned comparison approach to examine the compo- nents of the trial blocks $\times$ ability interaction, it should be noted that none of the above pairwise comparisons that were found to be nonsignificant by the very conservative Scheffé procedure were significant when using a more powerful planned comparison procedure. Thus, it is highly unlikely that any important differences were missed by using the Scheffé procedure. Consistent with Experiment 1 and other results, participants made more dimensional responses to the density triads $(M=12.82, S D=4.79)$ than to the length triads $(M=7.65, S D=5.21)$.

An analysis of variance conducted on the number of similarity responses revealed complementary results. Thus, it appears that the tendency to process information in a separable or integral fashion and to shift to that mode of processing with experience in the task is related to at least one measure of the ability to extract relevant components from an irrelevant background, that is, performance on the GEFT. Individual differences in processing stimulus dimensions do appear to make a difference, at least in the sense that they relate to this one measure. Furthermore, the differences are what one would anticipate on the basis of Garner's (1974) theoretical account of the difference between separable and integral perception. That is, separable processing appears to be associated with greater selective processing ability.

\section{EXPERIMENT 3}

The results of Experiment 2 stand in apparent conflict with those reported by J. D. Smith and Baron (1981). In the J. D. Smith and Baron study, there was no relation between restricted classification performance and a measure of selective filtering ability, whereas in Experiment 2 of the present study there was such a relation. There are at least three possible explanations for this discrepancy. First, different dimensions were employed in the different studies. It may be that relations between classifying behavior and selective attention hold for some dimensions but not for others. Second, the types of "filtering" tasks employed were quite different. J. D. Smith and Baron employed a speeded sorting task, whereas the GEFT was used in the present study (Experiment 2). It is possible that only certain measures of the ability to separate relevant from irrelevant information will be found to relate to classifying behavior. Finally, the results of Experiment 2 indicate that a shift toward dimensional responding over trial blocks may be the most important aspect of individual differences, at least as those differences relate to selective processing abilities. J. D. Smith and Baron's procedures did not allow a look at changes in responding across trial blocks. In order to test between these possibilities, J. D. Smith and Baron's procedures were followed very closely, using the dimensions of length and density as employed in Experiment 2 of the present series. That is, participants performed both a restricted classification task and a speeded sorting task with stimuli varying in length and density. The major procedural 
difference between this experiment and that reported by J. D. Smith and Baron is that changes in responding across trial blocks were observed in the present experiment.

\section{Method}

Participants. The participants were 64 undergraduates enrolled in introductory psychology classes at Texas A\&M University.

Stimuli and Procedure. The stimuli for the restricted classification task were the length-density triads described previously; they were presented according to procedures described for Experiment 1 . All participants performed the restricted classification task and then a speeded sorting task.

The stimuli for the speeded sorting task were 2- or 4-cm-long lines with .25 - or $.50-\mathrm{cm}$ interdot-distance density. The lines were typed individually onto index cards, and the cards were laminated to facilitate sorting.

In all of the sorting conditions, the participant was handed a deck of 24 cards and was instructed to sort each deck into two piles, one just to his or her left and one just to his or her right. The experimenter indicated how the sorting was to be done for each deck by placing example cards to the left and right of the participant and saying "put all of the ones like this here (pointing) and all of the ones like this here (pointing)." The participant was instructed to sort as quickly as he or she could without making mistakes. The experimenter timed each sort with a stopwatch and also recorded the number of errors for each sort.

For half of the sorts, length was the relevant dimension, and for the other half, density was the relevant dimension. In control conditions, the irrelevant dimension was held constant, whereas in the interference conditions, the irrelevant dimension varied orthogonally to the relevant dimension. Thus, the difference in sorting times between the control and interference conditions could be taken as a measure of the ability to "filter" the irrelevant dimension. "That is, to the extent that a participant has difficulty filtering the irrelevant dimension, variations in that dimension should interfere with sorting on the basis of the relevant dimension.

The decks for the length-relevant control condition contained 12 2 -cm-long lines and $124-\mathrm{cm}$-long lines, all with .25 -cm density. The density-relevant control decks contained 12 lines of $.25-\mathrm{cm}$ density and 12 lines of $.50-\mathrm{cm}$ density, all $4 \mathrm{~cm}$ long. Regardless of whether length or density was the relevant dimension for sorting, the interference deck always contained six each of the four possible combinations of the two lengths with the two densities.

The participants performed a total of 24 sorts, 12 with length relevant and 12 with density relevant. They performed all 12 sorts with one dimension relevant and then all 12 sorts with the other dimension relevant. Half sorted with length relevant first, and half sorted with density relevant first. Within each of the blocks of 12 sorts, the participants alternated between control and interference decks such that half of the sorts were with the control deck and half were with the interference deck. Half of the participants started each block of 12 sorts with a control deck and half started with the interference deck. The first four sorts with each dimension relevant were considered to be practice trials, and no data from those trials were included in the analyses reported below.

\section{Results and Discussion}

The mean sorting times (and standard deviations) for the density-relevant sorts were 15.38 (3.42) and 15.99 (3.46) seconds for the control and interference conditions, respectively. Mean times (and standard deviations) for the length-relevant sorts were $14.96(2.97)$ and $15.46(3.12)$ seconds for the control and interference conditions, repectively. A repeated measures analysis of variance was conducted on the sorting times with both condition (control vs. interference) and relevant dimension (length vs. density) as factors. The analysis revealed that, overall, the interference sorting times were longer than the control times $[F(1,50)=20.36, p<.001]$, and that this effect did not interact with the relevant dimension factor $(F<1)$. Thus, participants appear to have had difficulty in filtering the irrelevant dimension, whether that dimension was length or density. Errors were virtually nonexistent and were not considered in this or subsequent analyses.

Of greatest interest is the relation between overall separable versus integral processing, as revealed in the restricted classification task and performance in the filtering task. In addition, the results of Experiment 2 indicate that changes in dimensional responding across trial blocks in the restricted classification task should be examined in relation to performance in the filtering task. In order to examine changes in responding over trial blocks in a manner comparable to that used in Experiment 2, the participants were divided into groups based on whether they had shown more or less than the mean amount of interference from variations in the irrelevant dimension (i.e., showed relatively more or less selective processing ability). The performance of these groups on the restricted classification task was then compared in an analysis of variance that included trial blocks as a factor. However, since density has been found to be a more salient dimension for classification in the present and previous studies (Ward, 1980, 1983), it was of interest to determine if relatively greater ability to filter that dimension than to filter length is differentially related to the tendency to give more separable responses in the restricted classification task. Therefore, rather than simply being divided into two groups (more vs. less than the mean amount of interference), the participants were divided into four groups: those who showed more than the mean amount of interference from irrelevant variation in both dimensions, those who showed less than the mean amount of interference from irrelevant variation in both dimensions, those who showed more than the average amount of interference from length but less than the average amount of interference from density, and those who showed more than the mean amount of interference from density but less than the mean amount of interference from length. The division of participants into those four groups was accomplished using the rawscore sorting times. That is, an individual's sorting time in the interference condition was subtracted from his or her time in the control condition, and then the absolute value of the group mean difference between the interference and control conditions. was added to the result. This was done separately for length-relevant and densityrelevant sorts. If the resultant value was positive, the individual was judged as showing less than the mean amount of interference from the irrelevant dimension in question, whereas if the value was negative the individual was judged as showing relatively more interference from the irrelevant dimension in question. The number (and standard deviation) of dimensional repsonses given in the restricted classification task by individuals in each of the four resulting groups is depicted in Table 3 separately for Trial 
Table 3

Number (and Standard Deviation) of Dimensional Responses Made in Trial Blocks 1, 2, and 3 by Participants Who

Showed More Than the Mean Amount of Interference from Irrelevant Variation in Density, Length, Both Dimensions, or Neither Dimension

\begin{tabular}{lccc}
\hline Greater Than Mean & \multicolumn{3}{c}{ Trial Block } \\
Interference From: & 1 & 2 & 3 \\
\hline Density & 5.25 & 5.31 & 5.31 \\
& $(3.64)$ & $(3.61)$ & $(4.03)$ \\
Length & 5.38 & 5.38 & 6.54 \\
& $(2.81)$ & $(3.57)$ & $(2.85)$ \\
Both & 4.75 & 4.56 & 5.50 \\
& $(1.98)$ & $(2.73)$ & $(3.05)$ \\
Neither & 4.63 & 5.84 & 6.53 \\
& $(3.30)$ & $(3.56)$ & $(3.15)$ \\
\hline
\end{tabular}

Blocks 1, 2, and 3. An analysis of variance conducted on the number of dimensional responses revealed significant effects for trial blocks $[\mathrm{F}(2,120)=10.33, \mathrm{p}<.001]$ and dimension type $[\mathrm{F}(1,60)=21.37, \mathrm{p}<.001]$. These first two results are consistent with those of Experiments 1 and 2 in indicating that participants made more dimensional responses in later trial blocks and that there were more dimensional responses to density $(M=10.20, S D$ $=5.77)$ than to length $(\mathrm{M}=6.03, \mathrm{SD}=5.93)$.

The most important findings in the present analysis were that the groups did not differ significantly in the overall number of dimensional responses given $(F<1)$, but there was a significant group $\times$ trial blocks interaction $[\mathrm{F}(6,120)=2.34, \mathrm{p}<.05]$. As indicated in Table 3, the nature of the interaction is that the individuals who showed less than the mean amount of interference from irrelevant variations in both dimensions in the speeded sorting task (i.e., those highest in selective processing ability) were the ones who showed the largest increase in dimensional responding. Since many pairwise comparisons could be used to assess the nature of this interaction, the Scheffe method was used to control the Type I error rate across all possible comparisons. However, since the Scheffe is an extremely conservative test, a significance level of . 10 was chosen to determine the critical $F$ ratio. Using the 6 and 120 degrees of freedom associated with the interaction in question, an F of 1.82 is required for significance at the .10 level. This makes the critical $F$ for the pairwise comparisons within that interaction equal to (groups -1$) \times($ trials -1$) \times 1.82$ or 10.92 . Using the critical $\mathrm{F}$ and the compromise error term suggested by Winer (1971), none of the between-group comparisons were significant at any of the trial blocks. (None were significant by the more powerful planned comparison procedure either.) Using the same critical $F$ ratio to determine whether any of the groups showed significant changes in responding across trial blocks, it was found that only the group that exhibited less than the mean amount of interference from both dimensions showed a significant increase in dimensional responding from Trial Block 1 to Trial Block $3[F(1,120)=22.74]$. The increase shown by the group that exhibited more than the mean amount of interference only from length approached, but did not reach, statistical significance $[F(1,120)=5.74] .{ }^{5}$ Neither of the other groups showed significant changes in responding over Trial Blocks 1 to 3 using the Scheffé procedure or the more powerful planned comparison procedure [F $<1$ for the group exhibiting more than the mean amount of interference from density, and $F(1,120)=2.98$ for the group exhibiting more than the mean amount of interference from both dimensions]. The nature of the present group $\times$ trial blocks interaction, then, is particularly interesting in that it parallels so closely the nature of the "filtering" ability $\times$ trial blocks interaction found in Experiment 2. In both studies, the participants exhibiting the greatest selective processing ability were also the ones who showed the greatest amount of increase in dimensional responding in the restricted classification task. Once again, the results illustrate the importance of considering changes in responding over trials. In the present study, no relation between classifying behavior and speeded sorting performance would have been found if such changes had not been considered.

The absence of an overall group effect is consistent with the findings of J. D. Smith and Baron (1981) in that there appears to be little or no relation between filtering ability, as measured by speeded sorting, and overall classification performance in the restricted classification task. However, the presence of a group $\times$ trial blocks interaction, which parallels a similar interaction from Experiment 2 , indicates that there is a relation between separableintegral processing in the restricted classification task and filtering ability as measured by speeded sorting. The relation, however, is subtler than might have been anticipated. It occurs only when considering the tendency to shift toward dimensional responding with exposure to the dimensions. Since J. D. Smith and Baron (1981) did not report changes in responding over trials, it is impossible to determine if the present results are consistent with or at odds with their findings.

One suggestive finding from the present study deserves mention in that it may relate to our ability to find relations between classifying behavior and performance in selective processing tasks. Although the group that showed a high level of interference only from length did not exhibit a significant increase in dimensional responses over trials in the restricted classification task, the pattern of change over trials for that group was more similar to the group showing little interference from both dimensions than it was to the other interference groups. The finding becomes more interesting when we conceptualize this "length interference" group as comprising those who were better able to filter density (i.e., they showed less than the mean amount of interference from irrelevant variation in density and more than the mean amount from length). What makes this finding potentially interesting is that density is the more salient of the two dimensions as judged by participants' consistent preference for that dimension as a basis of classification in these and previous studies (Ward, 1980, 1983). It may be that people's 
ability to filter highly salient dimensions is more related to their separable-integral classifying style than is their ability to filter less salient dimensions. Future studies with other dimension pairs that vary in salience appear to be needed to investigate this issue further.

\section{GENERAL DISCUSSION}

The results of the present experiments advance our understanding of individual differences in processing dimensional information, suggest that those differences are related to certain processing abilities, and speak to the issue of convergent operations in identifying separable versus integral perception. Each of these aspects of the results will be considered separately below.

\section{Individual Differences}

It appears that there are reliable differences between individuals in the way they perceive and process stimulus dimensions, with some individuals employing primarily separable modes of processing and others employing primarily integral modes. Put differently, different individuals appear reliably and consistently to perceive the very same physical stimuli as different types of psychological stimuli. Previous studies have indicated that these differences obtain across dimensions classed as separable and integral and also across auditory and visual modalities (J. D. Smith \& Baron, 1981). The present results extend our knowledge of such differences in two ways.

First, the results of Experiment 1 indicate that individual differences in processing dimensional information generalize to dimensions that are moderately separable (i.e., length and density; see Ward, 1980, 1983) and separate (i.e., circle size and radius orientation; see Burns et al., 1978). This is more than just an extension of results to yet another set of dimensions. The stimuli employed were, theoretically, different in terms of the type of analysis required for the perception of their dimensional structures. Burns et al. (1978) suggest that the dimensional structure of circle size and radius orientation is perceived automatically. In contrast, Ward (1983) suggested that extended processing is required to break length-density stimuli into component dimensions, as evidenced by the fact that longer processing times were associated with higher levels of dimensional responding to such items. The fact that the tendency to give more or fewer dimensional responses generalizes to dimensions that require different amounts of analysis for the perception of their dimensional structures attests to the strength of the individual differences under investigation.

The second way in which the present results extend our knowledge is that they indicate that individuals differ not only in their overall tendency to process in a separable or integral manner, but also in the tendency to shift toward dimensional responding with experience in the task. This tendency contributes to, but does not completely determine, the observed individual differences in overall dimensional responding. That is, individuals do appear to come to the task with separable or integral styles of responding (significant correlations in Trial Block 1) and also to further differentiate those styles with experience (increases in correlations across trial blocks). It is interesting to note that the individual difference findings discussed in this section mimic developmental findings reported by Ward (1980). In those studies, adults showed higher levels of dimensional responding than did children and were also more likely to shift to such responses with experience in the task.

\section{Relation to Processing Abilities}

It appears that individual differences in separableintegral processing (as revealed by restricted classification performance) are related to some measures of selective processing ability. Thus, there is suggestive evidence that the individual differences that have been observed in restricted classification-task performance reflect more than differential responsiveness to the instructions or demands of one particular task. The relation is stronger when the tendency to shift to more dimensional responding across trials is considered than when just the overall amounts of separable-integral responding are considered. Individuals who show the largest increases in dimensional responding are those who perform most effectively on the GEFT and in speeded sorting tasks. The results are what one would anticipate from Garner's distinction between separable and integral perception. That is, selective attention is thought to be easier when dimensions are perceived separably, and a tendency to show increased separable responding appears to be associated with better selective processing ability. Thus, Garner's caveat regarding the importance of considering the stimulus in models of processing also applies to individual differences in the processing of stimulus dimensions.

One discrepancy between the results of Experiment 2 and those of Experiment 3 is that the overall tendency to give more or fewer dimensional responses correlated with GEFT performance but not with speeded sorting performance. The discrepancy may be more apparent than real in that the finding of a relation between overall dimensional responding and GEFT performance was based largely on the greater tendency of the high GEFT group to increase dimensional responding across trial blocks. Recall that the high and low GEFT groups did not differ on Trial Block 1. Thus, the most potent individual difference factor in both Experiment 2 and Experiment 3 appears to be the tendency to shift to more dimensional responding across trial blocks. These results illustrate the importance of examining changes in responding across trials in studies concerned with the processing of stimulus dimensions and individual differences in that processing.

One possible explanation of the difference between the results of Experiments 2 and 3 may be the different apparent task demands. In the speeded sorting task, participants had to rapidly sort stimuli on the basis of one dimension while ignoring variations in an irrelevant 
dimension. Even though both speed and accuracy were emphasized, the participants may have felt pressured to respond within particular time constraints. The GEFT is also timed, but the time pressure $(10 \mathrm{~min})$ may be less immediately obvious to the participant. Similarly, the time pressure in the restricted classification task is less obvious. Since responding within shorter time constraints leads to more integral responding (J.D. Smith \& Kemler-Nelson, 1984; Ward, 1983), the speeded sorting task may have caused all participants to shift to more integral modes of processing, thus reducing individual differences in that task. Such an effect would reduce the relation between speeded sorting performance and performance in the restricted classification task. Consistent with this interpretation is the fact that participants showed interference from the irrelevant dimension in the sorting task, a pattern more characteristic of integral dimensions, whereas they showed a preponderance of separable responses to the same dimensions in the restricted classification task.

Regardless of the ultimate explanation of the present findings, the findings themselves are important in that they demonstrate that the tendency to perceive integrally or separably is related to certain processing abilities. Individual differences in the restricted classification task do make a difference. It becomes important, therefore, to consider what other processing abilities or tendencies may be related to a separable versus integral perceptual tendency. For example, susceptibility to environmental context manipulations in memory studies (e.g., S. M. Smith, Glenburg, \& Bjork, 1978) may be related to an integral versus separable style. That is, individuals with an integral style of processing may fail to separate from the learning context the material to be learned and thus be strongly affected by context manipulations. Studies of this and similar issues could advance our understanding of both the areas in question and individual differences.

The individual differences discussed in the present paper may even bear on our understanding of the process involved in responding to clinical assessment devices. For example, Stagner and Ward (1983) found that individuals who gave many separable responses in the restricted classification task attempted more synthesis and gave more total and whole responses on the Rorschach inkblot test than did individuals who gave fewer separable responses.

\section{Convergent Operations}

When used in a between-subjects design, restricted classification and speeded sorting tasks converge on the distinction between separable and integral dimensions (Garner, 1974). That is, separable dimensions result in dimensional classifications, and little interference in sorting, whereas integral dimensions result in similarity classifications and much interference in sorting. However, in a within-subject design, convergence appears to break down somewhat. That is, individuals who give many dimensional classifications are not necessarily those who show little interference, at least in the overall sense.
Although the above pattern may pose problems for the separable-integral distinction, it may be as informative as it is problematic. The fact that convergence holds between subjects may indicate that, in fact, particular dimensions are either separable or integral relative to other dimensions and that those differences hold across tasks. The fact that convergence breaks down within subjects may indicate, as noted earlier, that different tasks place different time demands on individuals and that individuals may respond differentially to those demands. Finally, since convergence within subjects appears to increase if we focus on the filtering of highly salient dimensions and consider changes in responding over trials, salience and experience with the dimensions may be crucial to consider. If such interpretations hold, they suggest that our models of information processing must increase in complexity. To fully understand the processing of stimulus dimensions, we must begin to consider not only process, stimulus, task demand, practice, and individual difference factors, but also the possible interactions among those factors.

\section{REFERENCES}

Burns, B., Shepp, B. E., McDonough, D., \& Wiener-Ehrlich, W. K. (1978). The relation between stimulus analyzability and perceived dimensional structure. In G. H. Bower (Ed.), The psychology of learning and motivation (Vol. 12). New York: Academic Press.

FELFOLDY, G. L. (1974). Repetition effects in choice reaction time to multidimensional stimuli. Perception \& Psychophysics, 15, 453-459.

Fisher, M. A. (1977). Dimensional interaction in hyperactive children: Classification of computer-displayed stimuli. Bulletin of the Psychonomic Society, 10, 443-446.

GARNER, W. R. (1974). The processing of information and structure. Potomac, MD: Erlbaum.

GARNER, W. R. (1976). Interaction of stimulus dimensions in concept and choice processes. Cognitive Psychology, 8, 98-123.

GARNER, W. R. (1983). Asymmetric interactions of stimulus dimensions in perceptual information processing. In T. S. Tighe \& B. E. Shepp (Eds.), Perception, cognition, and development: Interactional analyses. Hillsdale, NJ: Erlbaum.

KEMLER, D. G. (1982). Classification in young and retarded children: The primacy of overall similarity relations. Child Development, 53, 768-779.

LOCKHEAD, G. R. (1966). Effects of dimensional redundancy on visual discrimination. Journal of Experimental Psychology, 72, 95-104.

Oltman, P. K., Raskin, E., \& Witkin, H. A. (1971). Group Embedded Figures Test. Palo Alto, CA: Consulting Psychologists Press.

SHEPARD, R. N. (1964). Attention and the metric structure of the stimulus space. Journal of Mathematical Psychology, 1, 54-87.

SHEPP, B. E., SwarTz, K. B. (1976). Selective attention and the processing of integral and nonintegral dimensions: A developmental study. Journal of Experimental Child Psychology, 22, 73-85.

Smith, J. D., \& Baron, J. (1981). Individual differences in the classification of stimuli by dimensions. Joumal of Experimental Psychology: Human Perception and Performance, 7, 1132-1145.

SMITH, J. D., \& KEMLER-NELSON, D. G. (1984). Overall similarity in adults' classifications: The child in all of us. Journal of Experimental Psychology: General, 113, 137-159.

SMITH, L. B., \& KEMLER, D. G. (1977). Developmental trends in free classification: Evidence for a new conceptualization of perceptual development. Journal of Experimental Child Psychology, 24, 279-298.

SMITH, S. M., GlenberG, A. M., \& BJoRK, R. A. (1978). Environmental context and human memory. Memory \& Cognition, 6, 342-353. 
STAGNER, B. H., \& WARD, T. B. (1983, August). The relation between cognitive processing and Rorschach responding. Paper presented at the meeting of the American Psychological Association, Anaheim, CA.

WARD, T. B. (1978). Dimensional responding in children and adults as a function of stimulus and response variables. Dissertation Abstracts International, 39, 4565B. (University Microfilms No. 78-23,091)

WARD, T. B. (1980). Separable and integral responding by children and adults to the dimensions of length and density. Child Development, 51, 676-684

WARD, T. B. (1983). Response tempo and separable-integral responding: Evidence for an integral-to-separable processing sequence in visual perception. Journal of Experimental Psychology: Human Perception and Performance, 9, 103-112.

WINER, B. J. (1971). Statistical principles in experimental design (2nd ed.). New York: McGraw-Hill.

Witkin, H. A., Oltman, P. K., Raskin, E., \& KarP, S. A. (1971). A manual for the Embedded Figures Tests. Palo Alto, CA: Consulting Psychologists Press.

\section{NOTES}

1. Although there may be other surface descriptions of the "dimensions" involved in constructing the circle stimuli, informal questioning of participants in the present study and more detailed questioning in other pilot studies indicates that the vast majority of participants view them as being composed of the two dimensions of size and radius orientation.

2 . The combination of length and density results in a third dimension, number. However, previous studies (Ward, 1980, 1983) indicate that number is not a salient dimension for classification in the present task. Therefore, only length and density triads were created.

3. Although the exact type of processing required for good performance on the GEFT may be open to debate, at its most basic level the task requires the participant to respond differentially to two components of a stimulus, the relevant simple figure and the irrelevant embedding context. The differential response may reflect selective attention, extraction of a signal from a noisy background, isolating of figure and ground, ignoring distraction, or some combination of these processes. Regardless of the exact processes involved, however, if the "separable" processor is viewed as being better able to selectively process the component parts of a stimulus, then that individual can be predicted to perform better on the GEFT than an "integral" processor who is less able to treat stimulus components independently.

4. Although there are alternative interpretations of such difference scores (see, e.g., Felfoldy, 1974), the task and interpretations used in the present study represent an attempt to be consistent with the approach of J. D. Smith and Baron (1981).

5. Note that the observed $F$ ratio would have been significant at the .05 level if the change from Trial Block 1 to Trial Block 3 had been a planned comparison. Since the $\mathrm{F}$ was significant by an overly powerful procedure and nonsignificant by a potentially overly conservative approach, it was compared with the value needed in a moderately conservative approach. Considering only the 12 trial block comparisons ( 3 comparisons for each of the 4 groups), the significance level needed to keep the overall Type I error rate at .05 is .004 (i.e., .05/12) using the modified least significant difference (lsd) approach (see Winer, 1971, p. 199). The $F(1,120)$ needed for significance at that level is approximately 8.18. Thus, the increase in dimensional responding for the group in question is not significant by the isd procedure either. The increase shown by the group that exhibited less than the mean amount of interference from both dimensions was significant, as determined by the planned comparison, Isd, or Scheffe procedure. Thus, we can be confident only of the significance of the increase shown by that group.

(Manuscript received August 31, 1984; revision accepted for publication April 4, 1985.) 